\title{
LOW-GRADE MYOFIBROBLASTIC SARCOMA OF THE LARYNX: CASE REPORT AND REVIEW OF LITERATURE
}

\author{
Petr Kordač , Dimitar Haď̆i Nikolov ${ }^{2}$, Katarína Smatanová ${ }^{1}$, David Kalfeřtt ${ }^{1}$
}

Department of Otorhinolaryngology and Head and Neck Surgery, University Hospital Hradec Králové, Charles University in Prague, Faculty of Medicine in Hradec Králové, Czech Republic ${ }^{1}$; The Fingerland Department of Pathology, University Hospital Hradec Králové, Charles University in Prague, Faculty of Medicine in Hradec Králové, Czech Republic ${ }^{2}$

Summary: Low-grade myofibroblastic sarcoma (LGMS) is a very rare, atypical myofibroblastic tumor with fibromatosis-like features with predilection mostly in head and neck region. LGMS occurs primarily in adult patients with a slight male predominance. Only few cases of LGMS affecting the larynx have been reported in literature to this date. We describe a case of low-grade myofibroblastic sarcoma of the larynx in a 40-year-old male patient. The clinicopathological characteristics, immunohistochemical findings and treatment are discussed.

Key words: Laryngeal tumor; Sarcoma; Low-grade myofibroblastic sarcoma

\section{Introduction}

Low-grade myofibroblastic sarcoma (LGMS) is a very rare, atypical myofibroblastic tumor with fibromatosis-like features with predilection mostly in head and neck region. LGMS occurs primarily in adult patients with a slight male predominance $(1,2)$. LGMS generally behaves as a lowgrade malignant sarcoma with a strong propensity to recur but only occasionally metastasise $(1,3,4)$. Although LGMS shows a wide anatomical distribution, it is most common in the head and neck region, especially in the tongue and oral cavity $(1,2,4-7)$. However, rare cases involving salivary glands, nasal cavity/paranasal sinuses, piriform fossa, parapharyngeal space and mandible have also been reported (8-12). Only few cases of LGMS affecting the larynx have been reported in literature to this date (13-17). We describe a case of low-grade myofibroblastic sarcoma of the larynx in a 40-year-old male patient. The clinic-pathological characteristics, immunohistochemical findings and treatment are discussed.

\section{Case report}

A 40-year-old male was examined in Department of Otorhinolaryngology and Head and Neck Surgery, University Hospital Hradec Králové, with 3 months history of hoarseness in April 2011. He was generally well in himself and a non-smoker. Videostroboscopy revealed redness of the right vocal cord with increased volume and visible capillaries on its surface without any vibratory function. There was a normal finding of the left vocal cord and both were moving symmetrically on phonation. Patient underwent di- rect microlaryngoscopy, where the epithelium layer of the right vocal cord was incised and in the deeper layers a white hard tissue was found and sent for histological examination. Histologically the tissue was verified as a pseudotumor with scarring and chronic inflammation. As low-grade mesenchymal lesion could not be excluded. Computed tomography of neck with contrast was performed. Vaguely opacificating hypotrophic infiltration of the right vocal cord without any significant cervical lymphadenopathy was found. Metastases were excluded thanks to further imaging (chest X-ray,

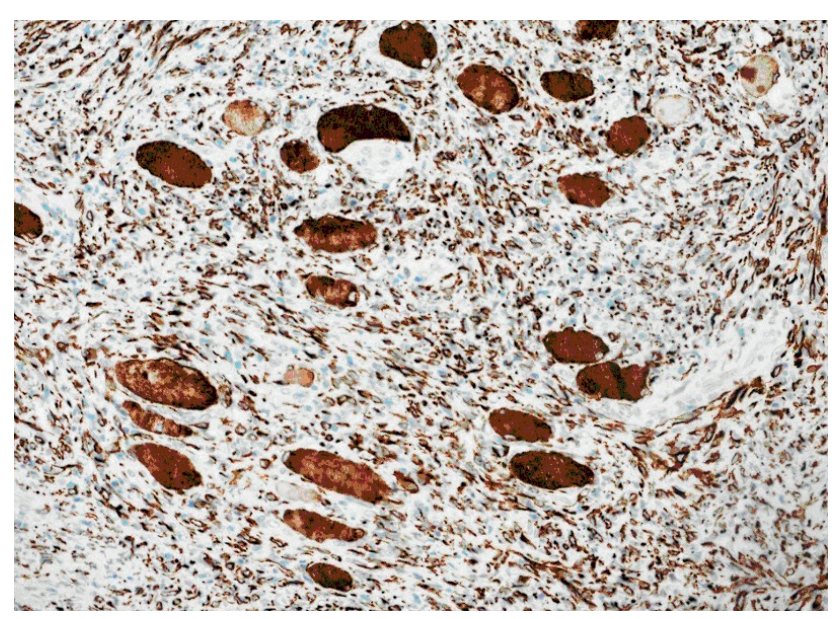

Fig. 1: Low-grade myofibroblastic sarcoma (blue color) infiltrating the adjacent muscle fibers (immunohistochemical staining for desmin, original magnification $200 \times$ ). 
abdominal ultrasound). In aim to remove the whole lesion from the paraglottic space (chordectomy) and repeat histological examination, surgical removal was performed via laryngofissure from external approach. The lesion had a hard consistency, localized under the intact surface of the vocal cord and extended to ventricle. Airway was secured with a temporary tracheostomy. Histology was confirmed low-grade myofibroblastic sarcoma infiltrating the adjacent muscle fibers now (Fig. 1).

As the boarders of the tumor were unknown, after discussion with oncologists, the residual soft tissue from the paralaryngeal space with inner perichondrium of thyroid cartilage (extended chordectomy with ventricular fold) were removed via laryngofissure. There was no malignancy found on histological examination. Radiotherapy was not indicated after the radical surgery. In early days after operation, tracheostomy was closed. The patient was discharged and is regularly followed up in our department. Site of the primary tumor is well visible on examination with flexible laryngoscope. The patient is without any suspicion of recurrence after 2 years (repeated flexible laryngoscopy, magnetic resonance imaging). He is well in himself, with no complaints; his voice is of good intensity, breathy on conversation.

\section{Discussion}

Sarcomas of larynx are exceedingly rare and account for less than $1 \%$ of malignant tumors of the head and neck region. Primary non-squamous tumors of the larynx are typically submucosal, derived mainly from mesenchymal cells, and account for approximately $0.3 \%$ to $1.0 \%$ of all laryngeal tumors (18).

Chondrosarcoma is the most common laryngeal sarcoma, but other types such as leiomyosarcoma, rhabdomyosarcoma, osteosarcoma, liposarcoma, and synovial sarcoma, have also been described (15). Sarcomas of the larynx may develop in any portion of the region. They are similar to carcinomas, however, they tend to originate in the vocal cords where they usually present as a painless submucosal mass (15). Associated symptoms depend on the size and location of the tumor, hoarseness and dyspnoea as most common.

Myofibroblastic differentiation is well described in sarcomas and is the hallmark of myofibroblastic sarcomas which can be either low or high grade. Histologically, the tumor cells of LGMS show diffuse fascicular or storiform growth pattern and they infiltrate surrounding tissues (2). The cytological and ultrastructural characteristics of neoplastic cells are consistent with myofibroblasts. Tumor cells in LGMS show a variable immunophenotype: actin positive or negative and desmin positive or negative. In our case was desmin positive. Tumor cells can express fibronectin, calponin, CD 34, CD 99 and CD 117, whereas S-100 protein, epithelial markers and h-caldesmon are negative $(2,4)$.

LGMS was first described by Mentzel et al. in 1998, who described a series of 18 sarcomas composed of cells with immuno-morphological features of myofibroblasts (4). Five LGMS arose in the oral cavity, four in the lower extremities, three in the upper extremities, four in the abdominal/ pelvic cavity, and two on the trunk (4). LGMSs have been reported to originate from many sites, but mostly develop in the soft tissues of the head and neck (1-3, 5, 7-12). LGMS mainly occurs in adults with a slight male predominance. The first two documented examples of laryngeal LGMS in the English literature was reported by MacGregor et al. in 2002 (13). Similarly as our case, in one patient was reported by MacGregor et al., the first biopsy diagnosis was benign lesion (laryngeal fibroma) (13).

The differential diagnosis of LGMS includes both benign and malignant lesions. Benign lesions include nodular fasciitis and fibromatosis, where both may display similar immunophenotype. Fibromatosis may be more hyalinised, may display a prominent nodularity, tends to infiltrate adjacent tissues, and atypia and mitotic figures are generally absent. Nodular fasciitis shows a heterogenous appearance with myxoid, cellular and fibrous areas. LGMS must be distinguished from leiomyosarcoma, which has fascicles of cells and blunt-ended or cigar-shaped nuclei and expresses h-caldesmon, while fibrosarcoma displays a herringbone pattern, high cellularity and prominent atypia $(12,17)$.

LGMS tends to have a relatively indolent course, showing frequent local recurrences and rare distant metastases $(4,20)$. Follow-up information is limited in the literature, but multiple soft tissue, bone, and pulmonary metastases were reported $(4,20)$. Although the treatment of LGMS has not been clearly defined, it may require surgical excision of the localised disease, radiotherapy of the primary site, or a combination of both modalities. Most cases were treated by local excision and some by wide excision. Few received additional radiotherapy and chemotherapy. Despite this, the role of postoperative radiotherapy and chemotherapy is controversial. Wide local excision and regular review are advocated by most authors $(1,4,5)$. The slow growth of the neoplasm is reflected in the late recurrence and small tumor size. The recurrent lesion did not show increased cellularity, proliferative activity, or increase in atypia compared with the primary tumor, unlike one metastatic case reported in the literature that was showing these features $(4,5)$.

For different laryngeal low-grade sarcomas, a laryngeal function preserving resection with narrow margins has been reported to be oncologically adequate (as opposed to a total laryngectomy) (21). Depending on the circumstances, large in individuals with reasonably functional voice, airway and/ or swallowing may best be managed by endoscopic debulking, as opposed to a partial laryngectomy. Even a local recurrence can potentially be treated with a salvage partial laryngectomy (21).

For example, Covello et al. reported case of laryngeal LGMS, in which the patient (69-year-old woman) underwent a supracricoid partial laryngectomy with cricohyoidoepiglottopexy and lymph node dissection was not performed. No further treatment was given and the patient remains free 
of disease 1 year after resection of the laryngeal tumor (15). However, one example of total laryngectomy for LGMS was reported by MacGregor et al. (13).

\section{Conclusion}

Our case report confirms the effectiveness of less radical surgery in order to excise the tumor with adjacent soft tissue aiming for negative margins, as it is not necessary for laryngeal LGMS for its relatively benign course. Despite this fact, we recommend long term follow-up. The role of postoperative radiotherapy or chemotherapy is controversial.

\section{Acknowledgements}

Supported by MH CZ-DRO (UHHK, 00179906).

\section{References}

1. Demarosi F, Bay A, Moneghini L, Carrassi A. Low-grade myofibroblastic sarcoma of the oral cavity. Oral Surg Oral Med Oral Pathol Oral Radiol Endod 2009; 108: $248-54$.

2. Laco J, Simakova E, et al. Low grade myofibroblastic sarcoma of tongue: a case report. Cesk Patol 2006; 42: 150-3

3. Yamada T, Yoshimura T, Kitamura N, Sasabe E, Ohno S, Yamamoto T. Low-grade myofibroblastic sarcoma of the palate. Int J Oral Sci 2012; 4: 170-3.

4. Mentzel T, Dry S, Katenkamp D, Fletcher CD. Low-grade myofibroblastic sarcoma: analysis of 18 cases in the spectrum of myofibroblastic tumors. Am J Surg Pathol 1998; 22: 1228-38.

5. Jay A, Piper K, Farthing PM, Carter J, Diwakar A. Low-grade myofibroblastic sarcoma of the tongue. Oral Surg Oral Med Oral Pathol Oral Radiol Endod 2007; 104: e52-8.

6. Montgomery E, Goldblum JR, Fisher C. Myofibrosarcoma: a clinicopathologic study. Am J Surg Pathol 2001; 25: 219-28.

7. Montebugnoli L, Venturi M, Gissi DB, Flamminio F, Foschini MP. Low-grade myofibroblastic sarcoma of the gingiva. BMJ Case Rep 2010.

8. Bisceglia M, Magro G. Low-grade myofibroblastic sarcoma of the salivary gland. Am J Surg Pathol 1999; 23: 1435-6.

9. Meng GZ, Zhang HY, Bu H, Yang GH, Zhang XL, Yang G. Myofibroblastic sarcoma of the nasal cavity and paranasal sinus: a clinicopathologic study of 6 cases and review of the literature. Oral Surg Oral Med Oral Pathol Oral Radiol Endod 2007; 104: 530-9.

10. Takahama A, Jr., Nascimento AG, Brum MC, Vargas PA, Lopes MA. Low-grade myofibroblastic sarcoma of the parapharyngeal space. Int J Oral Maxillofac Surg 2006; 35: 965-8.

11. Niedzielska I, Janic T, Mrowiec B. Low-grade myofibroblastic sarcoma of the mandible: a case report. J Med Case Rep 2009; 3: 8458

12. Coyne JD. Low-grade myofibroblastic sarcoma of the piriform fossa: a case report with a literature review of a tumour with a predilection for the head and neck. $\mathrm{Br}$ J Oral Maxillofac Surg 2007; 45: 335-7.

13. MacGregor AR, Batsakis JG, El-Naggar AK. Myofibroblastoma of the larynx: a study of two cases. Head Neck 2003; 25: 606-11.

14. Schroder S, Stengel B, Radtke A, Kleemann D. Myofibroblastic sarcoma of the larynx: a case report and review. HNO 2009; 57: 1311-6.

15. Covello R, Licci S, et al. Low-grade myofibroblastic sarcoma of the larynx. Int J Surg Pathol 2011; 19(6): 822-6.

16. Cheng KJ, Wang SQ, Zhou SH. Low-grade myofibroblastic sarcoma of the larynx: a case report. Zhonghua Er Bi Yan Hou Tou Jing Wai Ke Za Zhi 2010; 45: 868-9.

17. Ni C, Xu YY, Zhou SH, Wang SQ. Differential diagnosis of inflammatory myofibroblastic tumour and low-grade myofibroblastic sarcoma: two case reports with a literature review. J Int Med Res 2011; 39: 311-20.

18. Farhood AI, Hajdu SI, Shiu MH, Strong EW. Soft tissue sarcomas of the head and neck in adults. Am J Surg 1990; 160: 365-9.

19. Fisher C. Myofibroblastic malignancies. Adv Anat Pathol 2004; 11: 190-201.

20. Watanabe K, Ogura G, Tajino T, Hoshi N, Suzuki T. Myofibrosarcoma of the bone: a clinicopathologic study. Am J Surg Pathol 2001; 25: 1501-7.

21. Friedman AD, Burns JA, Lutch MJ, Zeitels SM. Submucosal neoplasms of the laryngeal introitus. J Laryngol Otol 2012; 126: 706-13.

Received: 03/09/2014

Corresponding author:

Accepted in revised form: 27/01/2015

David Kalfeřt, Department of Otorhinolaryngology and Head and Neck Surgery, University Hospital Hradec Králové, Sokolská 581, Hradec Králové 500 05, Czech Republic; e-mail: kalfertd@1fhk.cuni.cz 